\title{
ADAPTIVE PREDICTIVE EXPERT (ADEX) CONTROL: APPLICATION TO WASTE WATER TREATMENT PLANTS
}

\author{
J. Riesco ${ }^{1}$, J. Calvo ${ }^{2}$ and J. M. Martín-Sánchez ${ }^{3}$ \\ ${ }^{I}$ ADEX, S.L., Plaza Valle de la Jarosa 77, Madrid 28035 \\ ${ }_{2}^{2}$ Alfatec, Bravo Murillo 12, Madrid 28003 \\ ${ }^{3}$ Dept. Sistemas Energéticos, E.T.S.I. de Minas, Rios Rosas 21, Madrid 28003
}

\begin{abstract}
This paper presents the application of a new adaptive predictive expert (ADEX) control methodology to the biological phase of a residual water treatment plant. The ADEX methodology adds an expert block into the operation of previously known adaptive predictive control systems. This expert block, based on rules and in the evolution of the process variables, determines and/or modifies the operation of the driver block, control block and adaptive feedback mechanism of the previous art, in order to improve the performance, robustness and stability of the overall control system. The biological process has an inherent multivariable, time-varying and non-linear dynamic nature, and is submitted to stochastic and discontinuous perturbations, such as significant changes in the flow rate of residual water and its load of dirtiness. The integration of ADEX controllers within a control and optimization strategy, implemented in the local PLC, allowed the new control system to achieve a precise control of the oxygen levels and to optimize the process operation.
\end{abstract}

Keywords: Adaptive Control, Predictive Control, Expert Systems, Optimization, Computer Controlled Systems, Water Treatment, Waste Treatment.

\section{INTRODUCTION}

The technology of waste water treatment plants is well known in its different mechanical, physicochemical and biological phases. However, the biological phase presents important process control difficulties, due to the multivariable, non-linear and time-varying nature of this kind of process, which in addition is submitted to stochastic and discontinuous perturbations [1-2]. PID based control systems, applied so far, are not generally able to stabilize satisfactorily the critical variables of the biological purification process, which deteriorates its performance and, particularly, the quality of the treated water and the consumption of energy in the plant.

This paper presents the application of a new adaptive predictive expert (ADEX) control methodology to the biological phase of a waste water treatment plant, in San Fernando, Madrid (Spain). Section 2 introduces ADEX methodology, which is basically a new generation of adaptive predictive control (APC) [3-5], and its implementation through the local control system, a PLC5 from Rockwell Automation. The biological process is described in Section 3. ADEX controllers were applied within the context of a control and optimization strategy, designed to operate within the desirable range for the control variables and to optimize the process performance, which is described in Section 4. Section 5 presents trend curves of the critical process variables, which illustrate the control performance obtained by the previous PID based system; these results are compared with those obtained after the implementation of the new system. Section 6 presents the conclusions.

\section{ADEX METHODOLOGY AND ITS IMPLEMENTATION}

ADEX methodology integrates Adaptive Predictive Control (APC) and Expert Control, in order to provide a control solution able to apply expert control, when required, and adaptive predictive control, when possible, optimizing in this way the global process operation. ADEX combines APC with Expert control by defining domains of operation for each of them in an integrated setup. The evolution of process variables determines if APC or Expert control must be applied to the process according to the corresponding domain of operation.

The Adaptive Predictive (AP) domains are those in which the dynamic cause-effect relationship between the input and output process variables can, in real time, be identified by means of a time-varying model driven by an adaptive mechanism. In these domains APC can be applied and the process operation is optimized.

ADEX allows the application of expert control in certain domains of operation where manual control may provide a more robust and efficient control than APC. Operator experience is used to develop the rules imitating manual control intelligence. If an excursion occurs, expert domains drive the process variables back towards the AP domains, where APC is applied.

ADEX domains are a fundamental contribution of the new methodology over the APC prior art. The experimental

\section{U.S. Government Work Not Protected By U.S. Copyright}


results shown later in Section 5 demonstrate that they make APC performance extremely efficient and robust, i.e., practical. The block diagram of an ADEX controller is presented in Fig. 1.

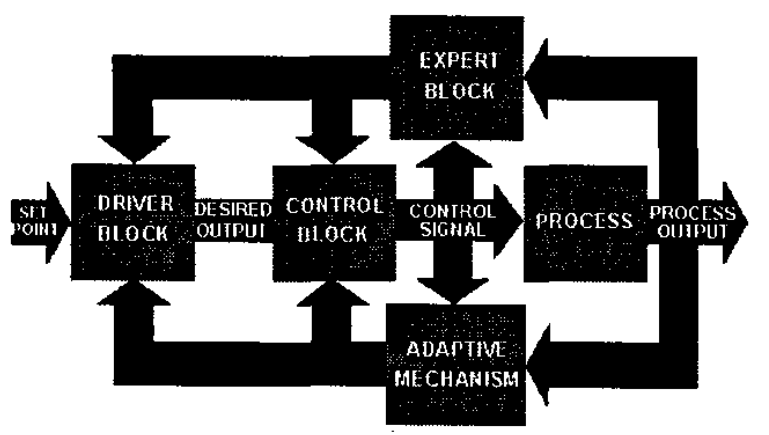

Figure 1. Block Diagram of an ADEX Controller

ADEX adds an expert block into the operation of previously known adaptive predictive control systems. This expert block, based on rules and in the evolution of the process variables, determines and/or modifies the operation of the driver block, control block and adaptive feedback mechanism of the previous art, in order to improve the performance, robustness and stability of the overall control system.

In this way, when the process $I / O$ variables evolve in a domain for adaptive predictive control, the expert block will determine the application of APC. The adaptive mechanism will identify the cause-effect relationship of the process and the control block will be able to predict and control the evolution of the process variables. On the other hand, when the process $\mathrm{I} / \mathrm{O}$ variables evolve in a domain for expert control, the expert block will determine the application of expert control. Then, the control block will compute the control vector based on rules that imitate the human operators intelligence, in a similar way as it is done in the well known fuzzy logic / expert systems.

ADEX technology was defined in an international patent application [6] and can be implemented through ADEX

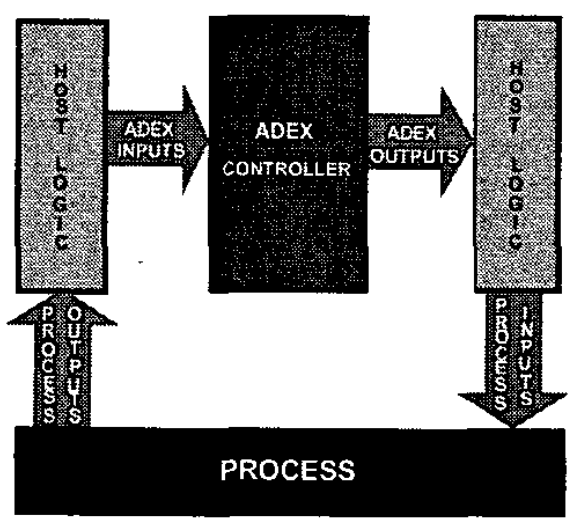

Figure 2. ADEX Controllers Integration within the Host System Control Logic
Control \& Optimization Platform (COP), which is a software platform that can be integrated with the control logic of the local control system (the host system). ADEX COP and its implementation are fully described in [7].

ADEX COP allows, as shown in Fig.2, the host system to integrate single-input single-output or multivariable $\mathrm{ADEX}$ controllers into its control logic, which can determine:

- How the process output signals have to be treated before being supplied to the ADEX controllers.

- How the ADEX controller outputs have to be treated before being applied to the process.

- The search for the operating points which optimize the process operation, taking advantage of the precise control and stability provided by the ADEX controllers.

Through the use of ADEX controllers, robust and precise control of the critical process variables and optimization of the process operation, is obtained. When the host system uses a graphical language to define its control logic, graphical ADEX operators will be integrated in the host library of operators for the graphical design of control \& optimization schematics.

\section{THE BIOLOGICAL PROCESS}

The biological process of the plant of San Fernando includes four pools fed independently by waste water coming from the mechanical and physicochemical phases of the plant. The flow rate of waste water entering each pool varies around 1.000 cubic meters per hour.

Pressurized air coming from a common pipe is introduced by the bottom of each pool and regulated by a butterfly valve in order to maintain a desirable concentration of oxygen in the pool. Under appropriate conditions of operation, a bacterial bloom eliminates the water impurities in the pools producing mud that is withdrawn from the bottom of each pool. To ensure the permanence of the bacterial blooms, a percentage of the flow rates of the evacuated mud is returned to the pools.

It is experimentally known that there is an optimum concentration of oxygen to reproduce the bacterial bloom that eliminates the water impurities. However, the flow rate of air required to attain this optimum level depends very much on the flow rate of waste water entering the pool, the load of impurity of the waste water and the state of the bacterial bloom itself.

Thus, control problems found in these kind of plants are due to the complex nature of the process, but also to the fact that is generally submitted to stochastic and discontinuous changes on the flow rate and load of impurity of the incoming waste water. Also, the evolution of the bacterial bloom itself continuously changes the load of impurity and oxygen level in each pool. 
On the other hand, since the air pressure in the common pipe affects the oxygen level in the pools, interactions between oxygen control in the different pools are present, since the control actions on the butterfly valves for each pool affect the pressure on the common air pipe.

Additionally, the real time information available on the state of the biological process is limited to the measurement of oxygen concentration in the pool. The oxygen sensor was located close to the pool outlet. Therefore, the process has an inherent multivariable, non-linear and time-varying dynamic nature, subjected to stochastic and discontinuous perturbations, and is a challenging application for any advanced control methodology.

\section{CONTROL \& OPTIMIZATION STRATEGY}

ADEX COP was integrated in the control logic of a PLC 5 from Rockwell Automation, which was already installed in the plant. ADEX controllers were applied to the biological process with the following control objectives: (i) to maintain oxygen concentrations in the pool at constant levels chosen by the operator, (ii) to maintain the air pressure in the pipe at levels that facilitate an optimal operation of the oxygen control loops and minimize the consumption of energy.

Thus, an ADEX controller was used to control the oxygen concentration level by acting on the butterfly valve position in each of the pools. Another ADEX controller was used to control the air pressure in the pipe by acting on the diffuser of the compressor. Three AP domains, centered around the set point, and two expert domains, were configured for each ADEX controller.

The control performance of the oxygen loops was facilitated when the corresponding butterfly valve positions remained in a range between $20 \%$ and $60 \%$ opening. Out of this range incremental control actions produced non-linear moderate increments in the air flow rate introduced into the pools. Taking into account these operating conditions, a control and optimization strategy was applied based on the following:

- When one of the butterfly valves gets over the upper threshold of $60 \%$ and the oxygen level in the corresponding pool is beyond 0.5 PPM under its set point, then the set point for the air pressure controller was increased by 1 millibar. This increase in the pipe air pressure was in order to produce a recovery in said oxygen level and, at the same time, a return of the valve position to the desired range of operation.

- On the other hand, when a butterfly valve gets under the lower threshold of $20 \%$ and the oxygen level in the corresponding pool is beyond 0.5 PPM over its set point, then the set point for the air pressure controller was decreased by 1 millibar, to obtain the desired opposed effect.

The local PLC control strategy was designed in such a way that, when the demand of air increased, due to a higher load of impurity in the incoming waste water, and the compressor diffuser reached its higher position, another onoff compressor would enter into operation to allow the supply of the required flow rate of air. When it happened, the air pressure controller had to deal with the start of the on-off compressor operation as a non measurable severe perturbation acting on the process. The plant had a back up diffuser controlled compressor and up to four on-off compressors, which could enter into operation if needed.

On the other hand, if the demand of air decreased and the compressor diffuser reached its lower position, an on-off compressor stopped operation.

The demand of air was variable during the day and from day to day, depending on many circumstances, such as the day of the week and the weather conditions. Sometimes the operation of the four on-off compressors was required.

\section{EXPERIMENTAL RESULTS}

The control system, previously used in the plant, considered PID loops to control the oxygen level in the biological purification pools and another PID loop to control the pressure in the air pipe. Based on practical experience, in order to improve the overall control performance of the plant, the actual set point on the pressure controller was changed around a certain desired value, taking into account the mean of the deviations of the oxygen measured in the pools with respect to their set point values.

The logic behind this strategy tried to use the air pressure in the pipe to reduce towards zero the mean of oxygen deviations in the pools, while using each oxygen PID loop to compensate for the particular oxygen deviation in the corresponding pool. This control strategy was found to be the best after a sound experimentation with various PID based control schemes. Typical results, obtained with this PID experimentally optimized control scheme are presented for comparative purposes with those obtained with the new system. The application of both systems were under similar operating conditions.

Fig. 3 shows 24 hours of PID control performance on the pressure of the common air pipe. The green (dark) line represents the air pressure evolution with time, while the white line represents the PID control action, i.e. the compressor diffuser position.

Fig. 4 shows 24 hours of PID control performance on the oxygen level at pool number 3 . The green (dark) line represents the oxygen evolution with time, which oscillates 
around the red straight set point line, which is at 3 PPM. The $Y$ axis presents a scale from 0 to 10 PPM of oxygen. The white line represents the PID control action, i.e. the butterfly valve opening. The results shown in Figs. 3 and 4 were considered satisfactory results under the operation of the plant previous control system and they illustrate its best control performance.

When the final control strategy for the new ADEX based control system was designed, implemented and ready to operate, a period of heavy rains made the oxygen content in the incoming water much higher than the desired set point for several days. Under these conditions although the ADEX control system was activated no significant control action was applied, since butterfly valves had to be closed and, the air pressure in the pipe was high in spite that the compressor diffuser was at its lower position.

Fig. 5 shows, with the same scales and variables of Fig.3, the starting of the ADEX pressure controller operation once the effect of the heavy rains disappeared. It may be observed that this happens about 14:58 of February 9, 2001,

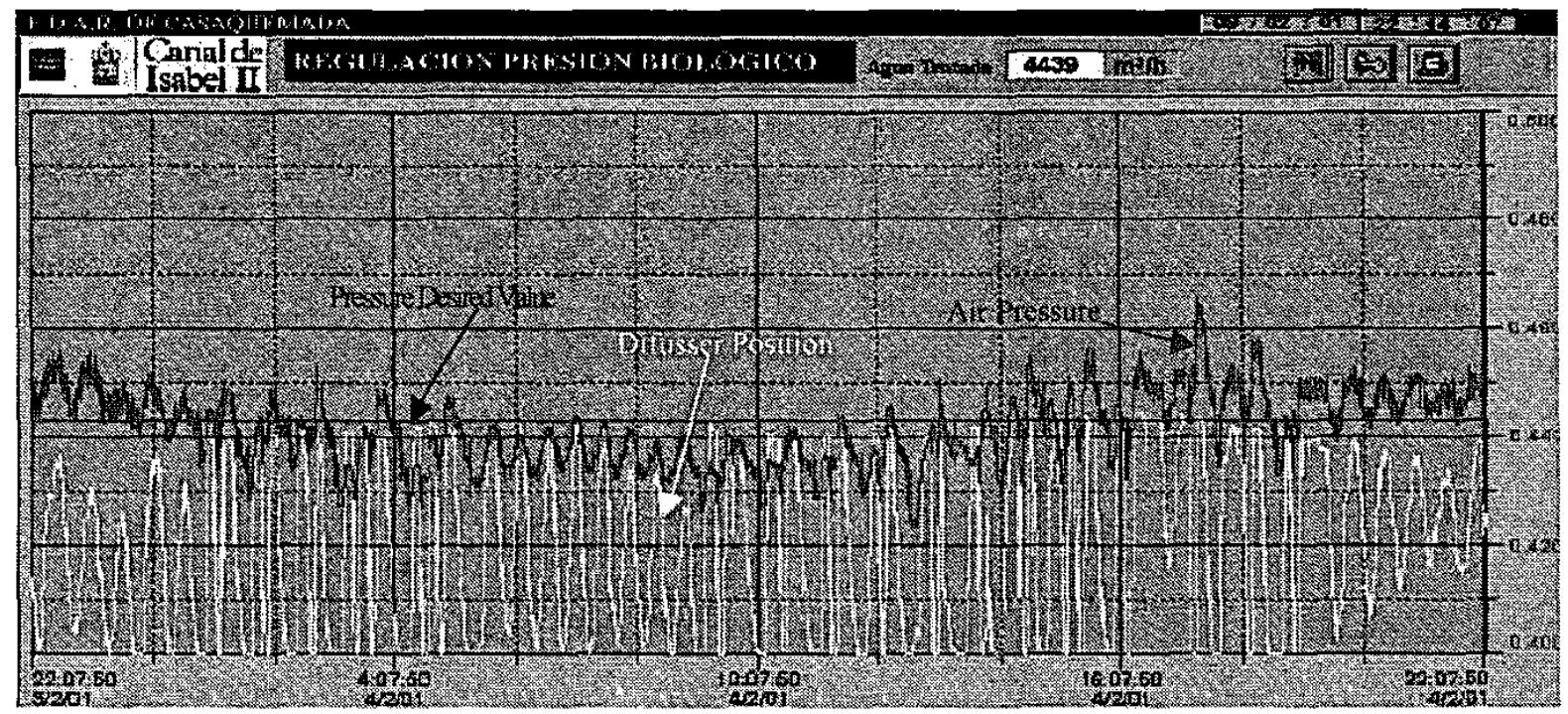

Figure 3. PID Control Performance on the Pressure of the Air Pipe

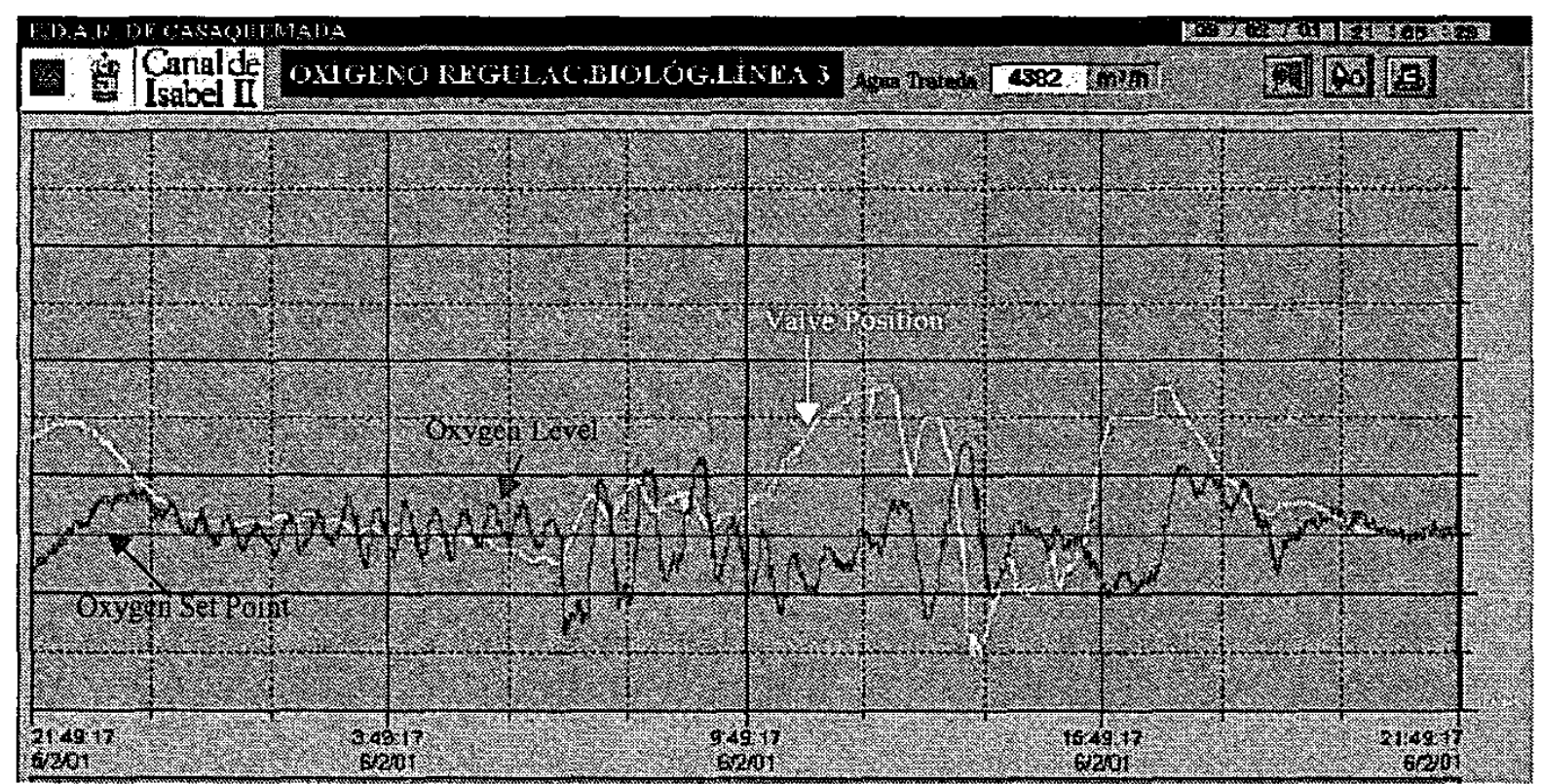

Figure 4. PID Control Performance (24 hours) on the Oxygen Level at Pool No. 3 
when the air pressure falls from a value higher than 0.48 to a value lower than 0.45 bar. Then, the ADEX pressure controller moves the compressor diffuser position from the lower position to the higher one. However, as the air pressure remains under its set point value, the PLC control logic starts the operation of an on-off compressor at about 15:30. It makes the ADEX controller to react by decreasing drastically the diffuser compressor position, in order to drive the air pressure to its set point. Once the air pressure reaches its set point, it may be seen how the ADEX controller has to progressively increase the diffuser position to maintain the air pressure on its set point. This is certainly due to a progressively higher demand of air by the process. Thus, the ADEX controller demonstrated a remarkable capacity to immediately obtain a precise and stable air pressure control.

Fig. 6 shows, with the same scales and variables of Fig. 4 , 24 hours of ADEX control performance of the oxygen level at the same pool number 3 and illustrates the control performance achieved by the new system.

Fig. 7 shows one hour evolution of the same variables of Fig. 6 for pool number 1, plus an additional variable which

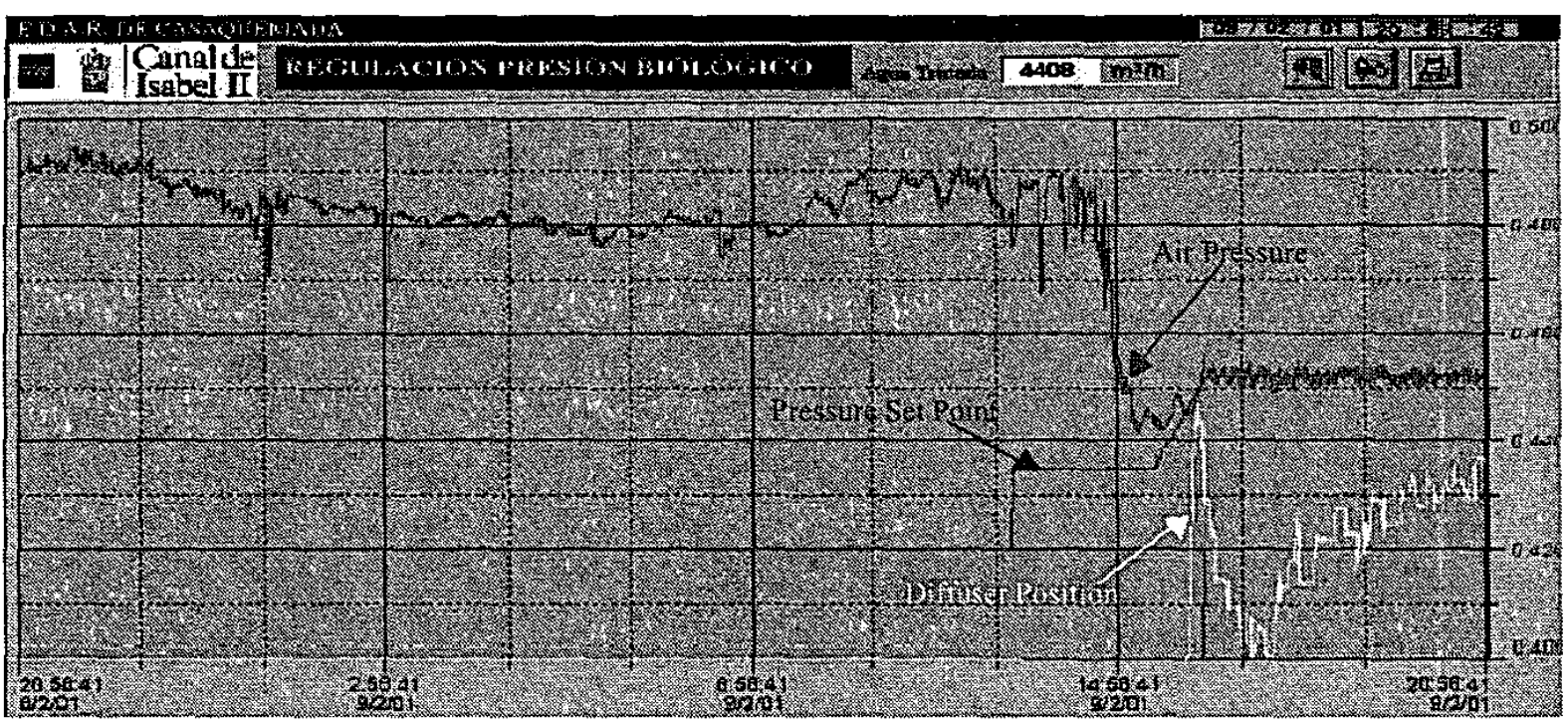

Figure 5. ADEX Control of the Air Pipe Pressure after a period of rains

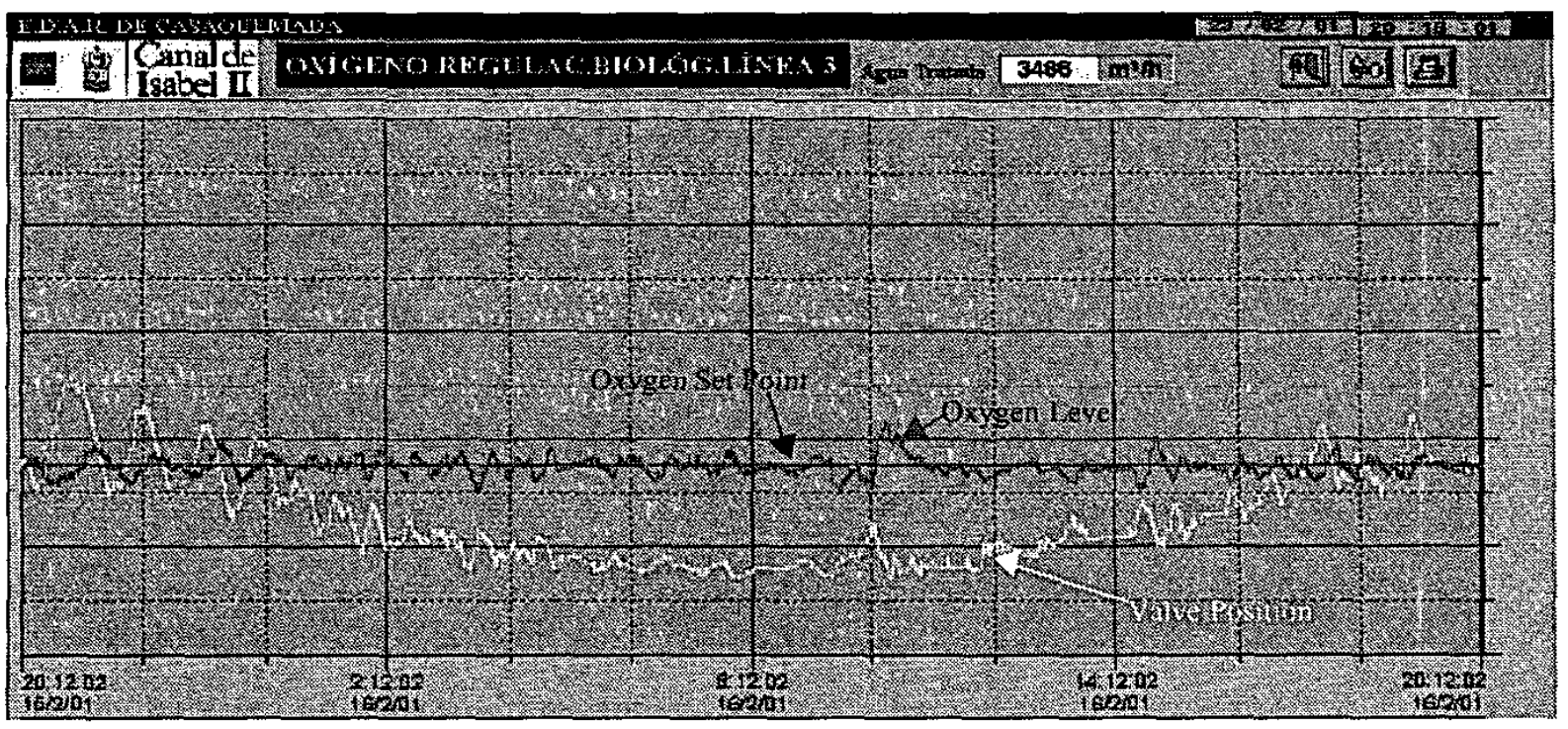

Figure 6. ADEX Control Performance (24 hours) on the Oxygen Level at Pool No. 3 
appears on the top of the graphic and represents the evolution of the air pipe pressure. It may be noticed that, about three minutes before $16: 10: 18$, the air pressure suffers a sudden increase and later on it returns in a soft and stable way to a slightly higher value.

This excursion on the air pressure has been produced by the start-up of an on-off compressor. The control action of the ADEX pressure controller, although not shown in Fig. 7, was able to stabilize again the air pressure on a desired value in less than three minutes. Thus, as it may be observed in Fig.7, the effect of such an important non measured perturbation acting on the process is compensated in such a way that it becomes practically unnoticed in the evolution of the oxygen level of the pool.

\section{CONCLUSIONS}

ADEX controllers were integrated within a control and optimization strategy, executed within the local PLC. This strategy varied the pipe air pressure set point taking into account the control signals on the butterfly valves and their desirable range of operation. Under this strategy the ADEX based control system achieved a precise and stable control of the critical variables of the biological process, in spite of the presence of significant, stochastic and discontinuous perturbations and its multivariable, non-linear and time varying dynamic nature.

The ADEX domains demonstrated to be an essential feature of the new methodology, improving the performance of the previous art [3-5] in terms of efficiency and robustness.

The standard deviation observed in the oxygen levels under the previous PID control system was always higher than 0.4 PPM, while the same standard deviation under the new control system is generally under 0.13 . Thus, oscillations of oxygen levels have been reduced to less than a third. This precise oxygen control was achieved with smooth control actions, improving the operating conditions of the biological process, the consumption of energy and the quality of the purified water.

\section{REFERENCES}

[1] "Biotechnology for the Treatment of Hazardous Waste". D.L. Stoner, Ed. Chelsea, MI. Lewis Publishers, 1994.

[2] C. Arnaiz, L. Isac and J. Lebrato, "Tratamiento biológico de aguas residuales", Tecnología del Agua, No. 198, March, 2000.

[3] J.M. Martín Sánchez, 'Adaptive Predictive Control System', USA Patent No. 4,197,576, 1976.

[4] J.M. Martín Sánchez, 'A New Solution to Adaptive Control', Proceedings of the IEEE, Special Issue on Adaptive Systems, August, 1976.

[5] J.M. Martín Sanchez and J. Rodellar, 'Adaptive Predictive Control: From the concepts to plant optimization', Prentice Hall, 1996.

[6] J.M. Martín Sánchez, 'Adaptive Predictive Expert Control System', International Patent Application. Application No: PCT/US00/17836, Priority Date: June 28, 2001.

[7] ADEX S.L.."ADEX Control and Optimization Platform: User Manual". Plaza Valle de la Jarosa, 77, Madrid, Spain, 2001.

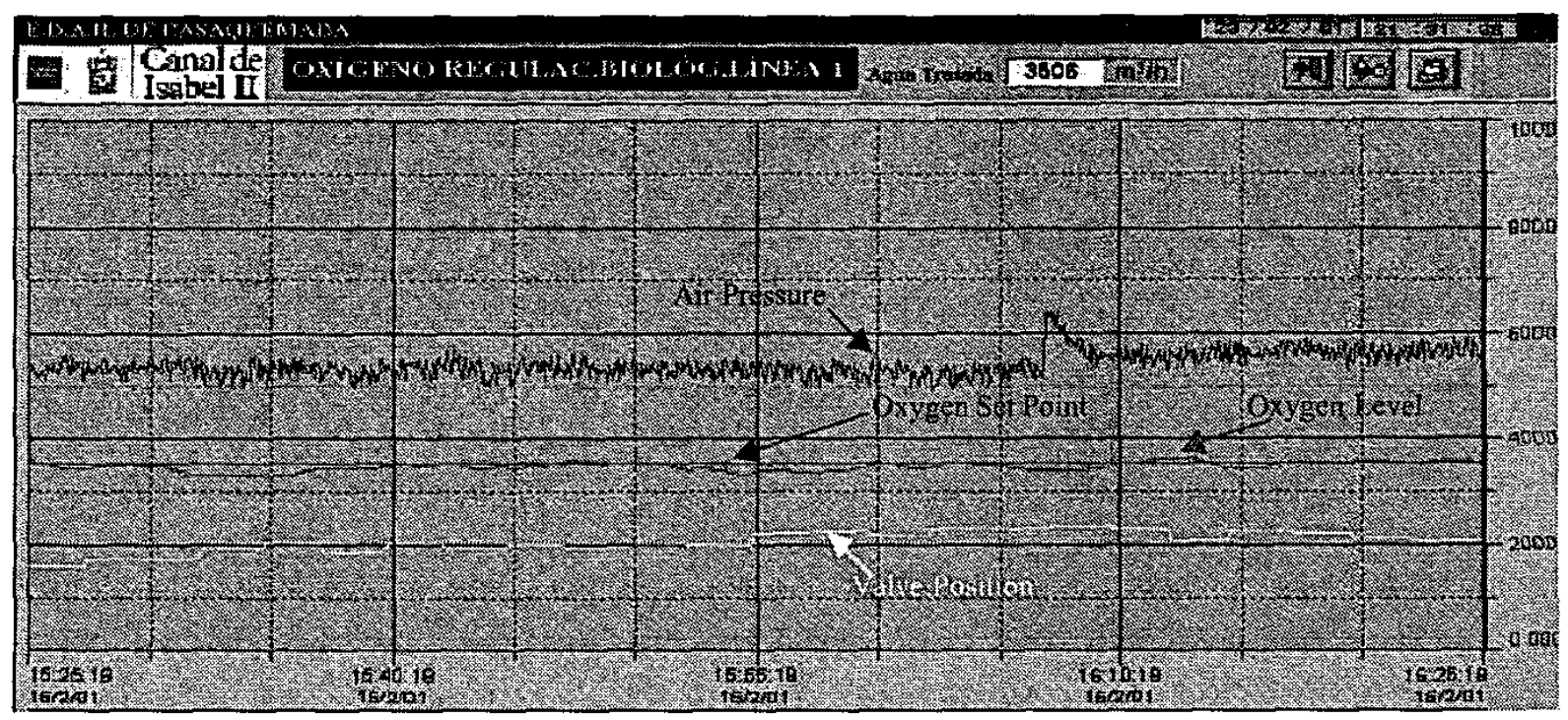

Figure 7. ADEX Control Performance (1 hour) in the presence of an On-Off Compressor Starting 